\title{
Research of Interference Elimination Method in Pollution with Fluorescent Bacterial
}

\author{
Jiang Weiwei ${ }^{1, a}$, Shan Jinbao ${ }^{2, a}$ \\ 1.Shandong Institute Of Commerce And Technology,Department of Food and Drug, Jinan Sh
andong 250103, China;
}

2.Shandong Institute Of Commerce And Technology, electronic information institute, Jinan Sh andong 250103, China

ajww_jiang@126.com

Keywords: interference elimination; fluorescent bacteria; pollution

\begin{abstract}
The method of interference elimination in pollution with fluorescent bacteria is researched. According to the interference of fluorescent bacteria in pollution, the interference elimination method in the pollution with fluorescent bacteria is proposed based on feature separation algorithm, the bacterial fluorescence signal is collected, $\mathrm{N}$ dimensional spatial direction vectors are constructed based on the signal, and the basic waveform of fluorescent bacterial signal is obtained, according to the basic waveform, the stretch and translation processing are taken, the objective function of fluorescent bacterial interference elimination in pollution is obtained, mathematical expectation of feature separation of fluorescent bacterial signal is calculated, and interference elimination of fluorescent bacterial pollution is realized. The experimental results show that this algorithm is applied in the fluorescent bacterial signal interference elimination, the anti interference performance is improved.
\end{abstract}

\section{Introduction}

With the increase of pollution, interference of pollution with fluorescent bacteria is obtained more and more attention. How to eliminate interfere in pollution with fluorescent bacterial has become a hot problem to research in the field of pollution, it has attracted wide attention of many experts and scholars[1]. At present, the interference fluorescent bacteria pollution elimination methods mainly include three kinds: interference elimination method based on optimal linear combination algorithm, the wavelet transform algorithm, and the collaborative optimization group algorithm, etc. the wavelet transform algorithm is most used in the fluorescent bacterial interference elimination. Because the fluorescent bacteria are widely applied in the pollution interference elimination, so it has the very big development prospect, and it has become the focus of many experts and scholars to study[2].

\section{Interference elimination principle of fluorescent bacteria in the pollution}

The fluorescent bacteria can be used to eliminate the interference in pollution, and the effective elimination method can reduce the interference of fluorescent bacteria of pollution, the interference elimination principle of fluorescent bacteria in the pollution is introduced firstly[3], the specific process of interference elimination is shown as follows:

Set $t(l)=\left\{t_{1}(l), t_{2}(l), \cdots, t_{p}(l)\right\}$ is the initial fluorescent bacterial signal, the signal are used to construct the $n$ dimensional space direction vectors, $y(l)=\left\{y_{1}(l), y_{2}(l), \cdots, y_{n}(l)\right\}$ is the $n$ dimension space numerical vectors, use the following formula to represent the original fluorescent bacterial acquisition signal:

$y(l)=B t(l)$

Assumed $\alpha(u) \in M^{2}(S), \alpha(\bar{\psi})$ is the wavelet of the wavelet transform process, assuming that the wavelet the wavelet conform to the following formula requirements[4-6], then the wavelet is called the basic wave. 
$D_{\alpha}=\int_{S} \frac{|\alpha(\alpha)|^{2}}{|\alpha|} d \alpha<\infty$

The basic wave is taken with the stretching and translation processing, the following formula can be obtained:

$\alpha_{c, d}(u)=\frac{1}{\sqrt{b}} \alpha\left(\frac{u-c}{b}\right), b, c \in S, u \neq 0$

The bacterial fluorescence signal is taken with the continuous wavelet transform processing, the signal is re-acquired and reconstructed, and the following formula is used to describe the process:

$g(u)=\frac{1}{D_{\alpha}} \int_{-\infty}^{\infty} \int_{-\infty}^{\infty} \frac{1}{b^{2}} X_{g}(b, c) \alpha\left(\frac{u-c}{a}\right) d b d c$

The objective function of fluorescent bacterial interference elimination in pollution is obtained, it can be calculated by the formula as follows:

$\frac{\partial H}{\partial Y}=\frac{2 Y}{X} E-\frac{2 Y}{W} \tilde{E}$

In the above formula, the extreme point of objective function $G(X, y)$ is zero point. Therefore, the formula as following can be obtained:

$X D=\frac{W}{V} X \tilde{D}$

In the above formula, $\hat{X}$ can be described by the feature component $\left(x_{1}, x_{2}, \cdots, x_{p}\right)$, assuming that these feature components and feature matrix $D$ is orthogonal relation, the feature matrix is mapped as $\tilde{D}$ with the orthogonal relation, the following results can be obtained:

$x_{j} \tilde{D} x_{k}^{U}=\sum_{v}\left[\tilde{z}_{j}(v)-z_{j}(v)\right]\left[\tilde{z}_{k}(v)-z_{k}(v)\right]=0$

$x_{j} D x_{k}^{U}=\sum_{v} z_{j}(v) z_{k}(v)=0, j, k=1,2, \cdots, p$ and $j \neq k$

Approximately, the fluorescent bacterial signal $\tilde{z}_{y}$ can be calculated as $\tilde{z}_{y} \approx z_{y-1}$, the following formula can be obtained:

$\left(z_{v}-\tilde{z}_{v}\right) \approx\left(z_{v}-z_{v-1}\right)=d z_{v}$

Assumed $e_{y}=1$, the following results can be calculated as:

$\left(z_{v}-\tilde{z}_{v}\right)=d z_{v} / d v=z^{\prime}$

The above results are taken into the following formula, the mathematical expectation of fluorescent bacterial signal elimination is:

$E\left[z_{j}^{\prime} z_{k}^{\prime}\right]=0, E\left[z_{j} z_{k}\right]=0$

According to the above formula we can learn that, as long as the fluorescence signal $z_{j}=x_{j} y$ is irrelevant to $z_{k}=x_{k} y$, and the derivative signal $z_{j}^{\prime}=x_{j} y^{\prime}$ is irrelevant, the extreme point of objective function can be obtained, according to $\hat{X}$, it can learn that fluorescent bacterial signal is statistical independent, according to the objective function, the interference of fluorescent bacterial pollution is eliminated.

\section{Experiment and results analysis}

In order to verify the validity of this algorithm, an experiment is taken. During the experiment, two different levels of interference are selected for experiment, the two interference coefficients are 0.45 , 
0.79. They respectively belong to two kinds of bacterial signal interference grads. When the interference coefficient is 0.45 , fluorescent bacterial signal interference elimination results of two algorithms are shown in Figure 1.

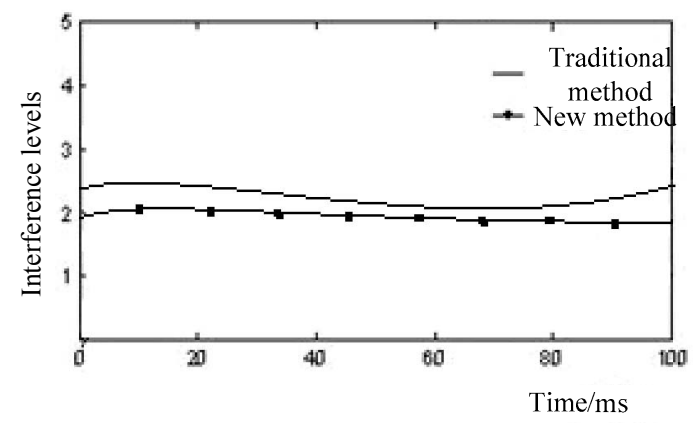

Figure 1. Interference elimination results when the interference coefficient is 0.45 When the interference coefficient is 0.79 , fluorescent bacterial signal interference elimination results of two algorithms are shown in Figure 2.

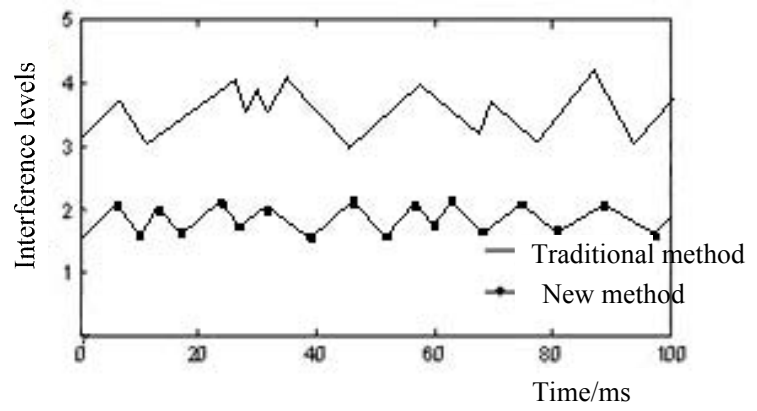

Figure 2. Fluorescent bacterial signal interference elimination results when coefficient is 0.79

On the basis of the above experiments, we can learn that, when the fluorescent bacterial signal interference intensity is weak, when the interference intensity is strong, the level of fluorescent bacterial signal interference signal eliminating result interference by using this method is slightly higher than the traditional algorithm. It shows that the anti interference performance is improved with new method.

\section{Conclusions}

In this paper, the method of interference elimination in pollution with fluorescent bacteria is researched. According to the interference of fluorescent bacteria in pollution, the interference elimination method in the pollution with fluorescent bacteria is proposed based on feature separation algorithm, the bacterial fluorescence signal is collected, $\mathrm{N}$ dimensional spatial direction vectors are constructed based on the signal, and the basic waveform of fluorescent bacterial signal is obtained, according to the basic waveform, the stretch and translation processing are taken, the objective function of fluorescent bacterial interference elimination in pollution is obtained, mathematical expectation of feature separation of fluorescent bacterial signal is calculated, and interference elimination of fluorescent bacterial pollution is realized. The experimental results show that this algorithm is applied in the fluorescent bacterial signal interference elimination, the anti interference performance is improved. This method will have good application value in practice.

\section{References}

[1] Rao Yutai,Yang fan. Network Intrusion Stir the Network Instability Control Methods of the Research[J]. BULLETIN OF SCIENCE AND TECHNOLOGY, 2014,30(1): 185-18 8 . 
[2] LI Feng, WU Chun- ming. Research on Prevention Fluctuation Control method of Net work Intrusion Based on Energy Management[J]. Computer simulation, 2013,30(12): 4548,335 .

[3] Alfaro V M, Vilanovab R. Robust tuning of 2DoF five-parameter PID controllers for in verse response controlled processes[J]. Journal of Process Control, 2013,23(4): 453-462

[4] Ou Shi-feng, Gao Ying, Zhao Xiao-hui. Adaptive Combination Algorithm and Its Modif ied Scheme for Blind Source Separation[J]. Journal of Electronics \& Information Techn ology, 2011, 33(5): 1243-1247.

[5] Xie S L, Liu Y, Yang J M, et al. Time-Frequency Approach to Underdetermined Bli nd Source Separation[J]. IEEE Transactions on Neural Networks and Learning System s, 2012, 23(2):306-315.

[6] XU Xiao-bin, ZHANG Guang-wei, SUN Qi-bo, et al. Precision Configurable Data Agg regation Algorithm in WSNs[J]. ACTA ELECT RONICA SINICA, 2014, 42(6):1205-120 9. 